\title{
AN OPTIMAL MATCHING PROBLEM FOR THE EUCLIDEAN DISTANCE
}

\author{
J. M. MAZÓN, J. D. ROSSI, AND J. TOLEDO
}

\begin{abstract}
We deal with an optimal matching problem, that is, we want to transport two measures to a given place, where they will match, minimizing the total transport cost that in our case is given by the sum of the Euclidean distance that each measure is transported. We show that such a problem has a solution. Furthermore we perform a method to approximate the solution of the problem taking limit as $p \rightarrow \infty$ in a system of PDE's of $p$-Laplacian type.
\end{abstract}

\section{INTRODUCTION}

We are interested in an optimal matching problem (see [6], [5]) that consists in transporting two commodities (say nuts and screws, we assume that we have the same total number of nuts and screws) to a prescribed location (say factories where we ensemble the nuts and the screws) in such a way that they match there (each factory receive the same number of nuts and of screws) and the total cost of the operation, measured in terms of the Euclidean distance that the commodities are transported, is minimized.

Optimal matching problems for uniformly convex cost where analyzed in [3], [5], [6] and have implications in economic theory (hedonic markets and equilibria), see [6], [7], [8], [9], [5] and references therein. However, when one considers the Euclidean distance as cost new difficulties appear since we deal with a non-uniformly convex cost.

Clearly, the optimal matching problem under consideration is related to the classical MongeKantorovich's mass transport problem. Using tools from this theory, we prove the existence of a solution of the optimal matching problem. We show that, in fact, the existence of solution is true changing the Euclidean norm by any norm in $\mathbb{R}^{N}$. Next, one of our main contributions in this paper is to perform a method to solve the problem taking limit as $p \rightarrow \infty$ in a system of PDE's of $p$-Laplacian type, which allows us to give more information about the matching measure and the Kantorovich potentials for the involved transport. This procedure to solve mass transport problems (taking limit as $p \rightarrow \infty$ in a $p$-Laplacian equation) was introduced by Evans and Gangbo in [12] and reveals quite fruitful, see [1], [16], [13]. We have to remark that the limit as $p \rightarrow \infty$ in the system requires some care since the system is nontrivially coupled and therefore the estimates for one component are related to the ones for the other, and we believe that it is interesting by its own.

Date: December 1, 2012. 
1.1. The optimal matching problem. To write the optimal matching problem in mathematical terms, we fix two non-negative compactly supported functions $f^{+}, f^{-} \in L^{\infty}$, with supports $X_{+}, X_{-}$, respectively, satisfying the mass balance condition

$$
M_{0}:=\int_{X_{+}} f^{+}=\int_{X_{-}} f^{-} .
$$

We also consider a compact set $D$ (the target set). Then we take a large bounded domain $\Omega$ such that it contains all the relevant sets, the supports of $f_{+}$and $f_{-}, X_{+}, X_{-}$and the target set $D$. For simplicity we will assume that $\Omega$ is a convex $C^{1,1}$ bounded open set. We also assume that

$$
X_{+} \cap X_{-}=\emptyset, \quad\left(X_{+} \cup X_{-}\right) \cap D=\emptyset \quad \text { and } \quad\left(X_{+} \cup X_{-}\right) \cup D \subset \subset \Omega .
$$

Whenever $T$ is a map from a measure space $(X, \mu)$ to an arbitrary space $Y$, we denote by $T \# \mu$ the pushforward measure of $\mu$ by $T$. Explicitly, $(T \# \mu)[B]=\mu\left[T^{-1}(B)\right]$. When we write $T \# f=g$, where $f$ and $g$ are nonnegative functions, this means that the measure having density $f$ is pushed-forward to the measure having density $g$.

For Borel functions $T_{ \pm}: \Omega \rightarrow \Omega$ such that $T_{+} \# f^{+}=T_{-} \# f^{-}$, we consider the functional

$$
\mathcal{F}\left(T_{+}, T_{-}\right):=\int_{\Omega}\left|x-T_{+}(x)\right| f^{+}(x) d x+\int_{\Omega}\left|y-T_{-}(y)\right| f^{-}(y) d y .
$$

The optimal matching problem can be stated as the minimization problem

$$
\min _{\left(T_{+}, T_{-}\right) \in \mathcal{A}_{D}\left(f^{+}, f^{-}\right)} \mathcal{F}\left(T_{+}, T_{-}\right),
$$

where

$$
\begin{array}{r}
\mathcal{A}_{D}\left(f^{+}, f^{-}\right):=\left\{\left(T_{+}, T_{-}\right): T_{ \pm}: \Omega \rightarrow \Omega \text { are Borel functions, } T_{ \pm}\left(X_{ \pm}\right) \subset D\right. \\
\left.\int_{T_{+}^{-1}(E)} f^{+}=\int_{T_{-}^{-1}(E)} f^{-} \text {for all Borel subset } E \text { of } \Omega\right\} .
\end{array}
$$

If $\left(T_{+}^{*}, T_{-}^{*}\right) \in \mathcal{A}_{D}\left(f^{+}, f^{-}\right)$is a minimizer of the optimal matching problem (1.1), we shall call the measure $\mu^{*}:=T_{+}^{*} \# f^{+}=T_{-}^{*} \# f^{-}$a matching measure to the problem. Note that there is no reason why a matching measure should be absolutely continuous with respect to the Lebesgue measure. In fact we shall see examples of matching measures that are singular (see Example 4.1).

We have the following existence theorem.

Theorem 1.1. The optimal matching problem (1.1) has a solution, that is, there exist Borel functions $\left(T_{+}^{*}, T_{-}^{*}\right) \in \mathcal{A}_{D}\left(f^{+}, f^{-}\right)$such that

$$
\mathcal{F}\left(T_{+}^{*}, T_{-}^{*}\right)=\inf _{\left(T_{+}, T_{-}\right) \in \mathcal{A}_{D}\left(f^{+}, f^{-}\right)} \mathcal{F}\left(T_{+}, T_{-}\right) .
$$


Moreover, we can obtain a solution $\left(\tilde{T}_{+}, \tilde{T}_{-}\right)$of the optimal matching problem (1.1) with a matching measure supported on the boundary of $D$.

Remark 1.2. We note that the fact that there is an optimal matching measure supported on $\partial D$ greatly simplifies the problem, since it allows to reduce the target set to its boundary.

For the quadratic cost function $c(x, y)=|y-x|^{2}$, the existence of a matching measure supported on the boundary of $D$ is not true in general, see [6].

We provide two different proofs to this existence theorem. The first one is more direct but does not provide a constructive way of getting the optimal matching measure in $D$, which is one of the unknowns in this problem; consequently, the construction of optimal transport maps (that are proved to exist) remains a difficult task. The main tool in this first proof is the use of ingredients from the classical Monge-Kantorovich theory. The second proof is by approximation of the associated Kantorovich potentials by a system of $p$-Laplacian type problems when $p$ goes to $\infty$. This approach provides an approximation of the potentials but also allows us to obtain the optimal measure in the limit. In addition we present several examples (that show that, in general, there is no uniqueness of the optimal configuration) and characterize when the optimal matching measure is a $\delta$.

Let us now introduce some notations, concepts and results from the Monge-Kantorovich Mass Transport Theory (see [1], [11], [17] and [18]) that will be used in the rest of the paper.

1.2. Monge-Kantorovich's Mass Transport Theory. Let $\Omega$ be an open bounded domain of $\mathbb{R}^{N}$. We denote by $\mathcal{M}(\Omega)$ the set of all Radon measures on $\Omega$ and by $\mathcal{M}^{+}(\Omega)$ the non-negative elements of $\mathcal{M}(\Omega)$. Given $\mu, \nu \in \mathcal{M}^{+}(\Omega)$ satisfying the mass balance condition

$$
\mu(\Omega)=\nu(\Omega)
$$

we denote by $\mathcal{A}(\mu, \nu)$ the set of transport maps pushing $\mu$ to $\nu$, that is, the set of Borel maps $T: \Omega \rightarrow \Omega$ such that $T \# \mu=\nu$. In the case $\mu=f \mathcal{L}^{N}\left\llcorner\Omega\right.$ and $\nu=g \mathcal{L}^{N}\llcorner\Omega$, we shall write $\mathcal{A}(f, g)$.

The Monge problem. Given $\mu, \nu \in \mathcal{M}^{+}(\Omega)$ satisfying the mass balance condition (1.2). The Monge problem, associated with the measures $\mu$ and $\nu$, is to find a map $T^{*} \in \mathcal{A}(\mu, \nu)$ which minimizes the cost functional

$$
\tilde{\mathcal{F}}(T):=\int_{\Omega}|x-T(x)| d \mu(x)
$$

in the set $\mathcal{A}(\mu, \nu)$. A map $T^{*} \in \mathcal{A}(\mu, \nu)$ satisfying $\tilde{\mathcal{F}}\left(T^{*}\right)=\min \{\tilde{\mathcal{F}}(T): T \in \mathcal{A}(\mu, \nu)\}$, is called an optimal transport map of $\mu$ to $\nu$.

In general, the Monge problem is ill-posed. To overcome the difficulties of the Monge problem, in 1942, L. V. Kantorovich ([14]) proposed to study a relaxed version of the Monge problem and, what is more relevant here, introduced a dual variational principle. 
Let us define $\pi_{t}(x, y):=(1-t) x+t y$. Given a Radon measure $\gamma$ in $\Omega \times \Omega$, its marginals are defined by $\operatorname{proj}_{x}(\gamma):=\pi_{0} \# \gamma, \operatorname{proj}_{y}(\gamma):=\pi_{1} \# \gamma$.

The Monge-Kantorovich problem. Fix $\mu, \nu \in \mathcal{M}^{+}(\Omega)$ satisfying the mass balance condition (1.2). The Monge-Kantorovich problem is the minimization problem

$$
\int_{\Omega \times \Omega}|x-y| d \gamma^{*}(x, y)=\min \left\{\int_{\Omega \times \Omega}|x-y| d \gamma(x, y): \gamma \in \Pi(\mu, \nu)\right\}
$$

where $\Pi(\mu, \nu):=\left\{\right.$ Radon measures $\gamma$ in $\left.\Omega \times \Omega: \pi_{0} \# \gamma=\mu, \pi_{1} \# \gamma=\nu\right\}$. The elements $\gamma \in$ $\Pi(\mu, \nu)$ are called transport plans between $\mu$ and $\nu$, and a minimizer $\gamma^{*}$ an optimal transport plan. These minimizers always exist.

The Monge-Kantorovich problem has a dual formulation that can be stated in this case as follows (see for instance [17, Theorem 1.14]).

Kantorovich-Rubinstein Theorem. Let $\mu, \nu \in \mathcal{M}(\Omega)$ be two measures satisfying the mass balance condition (1.2). Then,

$$
\min \left\{\int_{\Omega \times \Omega}|x-y| d \gamma(x, y): \gamma \in \Pi(\mu, \nu)\right\}=\sup \left\{\int_{\Omega} u d(\mu-\nu): u \in K_{1}(X)\right\}
$$

where $K_{1}(\Omega):=\{u: X \rightarrow \mathbb{R}:|u(x)-u(y)| \leq|x-y| \quad \forall x, y \in \Omega\}$ is the set of 1-Lipschitz functions in $\Omega$.

The maximizers $u^{*}$ of the right hand side of (1.3) are called Kantorovich potentials.

Let us briefly summarize the contents of this paper. Section 2 is devoted to the proof of Theorem 1.1; in Section 3 we study the limit as $p \rightarrow \infty$ in a $p$-Laplacian system obtaining more information about the solution of the matching problem; in Section 4 we describe some examples and characterize the geometrical configurations for which the matching measure is a point mass, finally, in Section 5 we collect final remarks.

\section{Proof of Theorem 1.1}

Let us denote by

$$
\mathcal{M}\left(D, M_{0}\right):=\left\{\mu \in \mathcal{M}^{+}(\Omega): \operatorname{supp}(\mu) \subset D, \mu(\Omega)=M_{0}\right\}
$$

the set of all possible matching measures. Given $\mu \in \mathcal{M}\left(D, M_{0}\right)$, we can consider the following minimization problem

$$
\inf _{\mu \in \mathcal{M}\left(D, M_{0}\right)} \inf _{\left(T_{+}, T_{-}\right) \in \mathcal{A}\left(f^{+}, f^{-}, \mu\right)} \mathcal{F}\left(T_{+}, T_{-}\right),
$$

where

$$
\mathcal{A}\left(f^{+}, f^{-}, \mu\right):=\left\{\left(T_{+}, T_{-}\right): T_{+} \in \mathcal{A}\left(f^{+}, \mu\right), T_{-} \in \mathcal{A}\left(f^{-}, \mu\right)\right\} .
$$

We have that, in fact,

$$
\inf _{\mu \in \mathcal{M}\left(D, M_{0}\right)} \inf _{\left(T_{+}, T_{-}\right) \in \mathcal{A}\left(f^{+}, f^{-}, \mu\right)} \mathcal{F}\left(T_{+}, T_{-}\right)=\inf _{\left(T_{+}, T_{-}\right) \in \mathcal{A}_{D}\left(f^{+}, f^{-}\right)} \mathcal{F}\left(T_{+}, T_{-}\right) .
$$


Indeed, observe that given $\left(T_{+}, T_{-}\right) \in \mathcal{A}_{D}\left(f^{+}, f^{-}\right)$, if we define

$$
\mu(E):=\int_{T_{+}^{-1}(E)} f^{+},
$$

we have that $\mu \in \mathcal{M}\left(D, M_{0}\right)$ and $\left(T_{+}, T_{-}\right) \in \mathcal{A}\left(f^{+}, f^{-}, \mu\right)$.

Note that in (2.1) we are considering all possible measures supported in $D$ with total mass $M_{0}$ and then we minimize the total transport cost. This is probably the most natural way of looking at the optimal matching problem and, as shown above, it is equivalent to our previous formulation.

We can see the optimal matching problem (2.1) as a kind of Monge's problem (recall the results gathered in the previous section). The corresponding Monge-Kantorovich's problem is the following

$$
\inf _{\mu \in \mathcal{M}\left(D, M_{0}\right)} \inf _{\left(\gamma_{+}, \gamma_{-}\right) \in \Pi\left(f^{+}, f^{-}, \mu\right)} \int_{\Omega \times \Omega}|x-y| d \gamma_{+}+\int_{\Omega \times \Omega}|x-y| d \gamma_{-},
$$

where $\Pi\left(f^{+}, f^{-}, \mu\right):=\left\{\left(\gamma_{+}, \gamma_{-}\right) \in \mathcal{M}^{+}(\Omega \times \Omega)^{2}: \gamma_{+} \in \Pi\left(f^{+}, \mu\right), \gamma_{-} \in \Pi\left(f^{-}, \mu\right)\right\}$. For this problem, similarly to $(2.2)$, we have that

$$
\begin{gathered}
\inf _{\mu \in \mathcal{M}\left(D, M_{0}\right)} \inf _{\left(\gamma_{+}, \gamma_{-}\right) \in \Pi\left(f^{+}, f^{-}, \mu\right)} \int_{\Omega \times \Omega}|x-y| d \gamma_{+}+\int_{\Omega \times \Omega}|x-y| d \gamma_{-} \\
=\inf _{\left(\gamma_{+}, \gamma_{-}\right) \in \Pi_{D}\left(f^{+}, f^{-}\right)} \int_{\Omega \times \Omega}|x-y| d \gamma_{+}+\int_{\Omega \times \Omega}|x-y| d \gamma_{-},
\end{gathered}
$$

where

$$
\Pi_{D}\left(f^{+}, f^{-}\right)=\left\{\left(\gamma_{+}, \gamma_{-}\right) \in \mathcal{M}^{+}(\Omega \times \Omega)^{2}: \pi_{0} \# \gamma_{ \pm}=f^{ \pm}, \pi_{1} \# \gamma_{+}=\pi_{1} \# \gamma_{-}, \operatorname{supp}\left(\pi_{1} \# \gamma_{ \pm}\right) \subset D\right\}
$$

Proof of Theorem 1.1. For a fixed $\mu \in \mathcal{M}\left(D, M_{0}\right)$, it is well known (see for instance [1]) that there exist Kantorovich potentials $u_{ \pm, \mu} \in W^{1, \infty}(\Omega)$, depending on $\mu$, with $\left|\nabla u_{ \pm, \mu}\right|_{\infty} \leq 1$, such that

$$
\begin{aligned}
\int_{\Omega} u_{+, \mu}\left(f^{+}-\mu\right) & =\max _{u \in W^{1, \infty}(\Omega),|\nabla u|_{\infty} \leq 1} \int_{\Omega} u\left(f^{+}-\mu\right)=\min _{T \in \mathcal{A}\left(f^{+}, \mu\right)} \int_{\Omega}|x-T(x)| f^{+}(x) d x \\
& =\min _{\gamma \in \Pi\left(f^{+}, \mu\right)} \int_{\Omega \times \Omega}|x-y| d \gamma
\end{aligned}
$$

and

$$
\begin{aligned}
\int_{\Omega} u_{-, \mu}\left(f^{-}-\mu\right) & =\max _{u \in W^{1, \infty}(\Omega),|\nabla u|_{\infty} \leq 1} \int_{\Omega} u\left(f^{-}-\mu\right)=\min _{T \in \mathcal{A}\left(f^{-}, \mu\right)} \int_{\Omega}|x-T(x)| f^{-}(x) d x \\
& =\min _{\gamma \in \Pi\left(f^{-}, \mu\right)} \int_{\Omega \times \Omega}|x-y| d \gamma .
\end{aligned}
$$


Therefore,

$$
\begin{gathered}
\sup _{\substack{v, w \in W^{1, \infty}(\Omega) \\
|\nabla v|_{\infty},|\nabla w|_{\infty} \leq 1}} \int_{\Omega} v f^{+}-w f^{-}+(w-v) \mu=\inf _{\left(T_{+}, T_{-}\right) \in \mathcal{A}\left(f^{+}, f^{-}, \mu\right)} \mathcal{F}\left(T_{+}, T_{-}\right) \\
\quad=\inf _{\left(\gamma_{+}, \gamma_{-}\right) \in \Pi\left(f^{+}, f^{-}, \mu\right)} \int_{\Omega \times \Omega}|x-y| d \gamma_{+}+\int_{\Omega \times \Omega}|x-y| d \gamma_{-} .
\end{gathered}
$$

Hence, from (2.2) and (2.3), we get

$$
\begin{aligned}
W:=\inf _{\mu \in \mathcal{M}\left(D, M_{0}\right)} & \sup _{\substack{v, w \in W^{1, \infty}(\Omega) \\
|\nabla v|_{\infty},|\nabla w|_{\infty} \leq 1}} \int_{\Omega} v f^{+}-w f^{-}+(w-v) \mu=\inf _{\left(T_{+}, T_{-}\right) \in \mathcal{A}_{D}\left(f^{+}, f^{-}\right)} \mathcal{F}\left(T_{+}, T_{-}\right) \\
= & \inf _{\left(\gamma_{+}, \gamma_{-}\right) \in \Pi_{D}\left(f^{+}, f^{-}\right)} \int_{\Omega \times \Omega}|x-y| d \gamma_{+}+\int_{\Omega \times \Omega}|x-y| d \gamma_{-} .
\end{aligned}
$$

Now, it is easy to see that there exists $\mu_{\infty} \in \mathcal{M}\left(D, M_{0}\right)$ such that

$$
\inf _{\mu \in \mathcal{M}\left(D, M_{0}\right)} \sup _{\substack{v, w \in W^{1, \infty}(\Omega) \\|\nabla v|_{\infty},|\nabla w|_{\infty} \leq 1}} \int_{\Omega} v f^{+}-w f^{-}+(w-v) \mu=\sup _{\substack{v, w \in W^{1, \infty}(\Omega) \\|\nabla v|_{\infty},|\nabla w|_{\infty} \leq 1}} \int_{\Omega} v f^{+}-w f^{-}+(w-v) \mu_{\infty} .
$$

Hence, by (2.4) for $\mu=\mu_{\infty}$ we get that

$$
W=\inf _{\left(T_{+}, T_{-}\right) \in \mathcal{A}\left(f^{+}, f^{-}, \mu_{\infty}\right)} \mathcal{F}\left(T_{+}, T_{-}\right)=\inf _{\left(\gamma_{+}, \gamma_{-}\right) \in \Pi\left(f^{+}, f^{-}, \mu_{\infty}\right)} \int_{\Omega \times \Omega}|x-y| d \gamma_{+}+\int_{\Omega \times \Omega}|x-y| d \gamma_{-} .
$$

Now, the proof of existence of optimal transport maps for the matching problem follows by $[1$, Theorem 6.2] which states the existence of an optimal transport map $T_{+}^{*}$ transferring $f^{+}$to $\mu_{\infty}$, and an optimal transport map $T_{-}^{*}$ transferring $f^{-}$to $\mu_{\infty}$.

Now, let us show that in our optimal matching problem we can restrict ourselves to measures supported on $\partial D$. Let us consider a minimizer $\left(T_{+}^{*}, T_{-}^{*}\right)$ of the matching problem and $h_{\infty}=$ $T_{+}^{*} \# f_{+}$the corresponding matching measure. Let us see that we can obtain a matching measure supported on $\partial D$. For $x \in \operatorname{supp}\left(f^{+}\right)$, let

$$
\alpha(x):=\min \left\{\alpha \in[0,1]:(1-\alpha) x+\alpha T_{+}^{*}(x) \in D\right\} .
$$

We define

$$
\tilde{T}_{+}(x):=(1-\alpha(x)) x+\alpha(x) T_{+}^{*}(x),
$$

that is, $\tilde{T}_{+}(x)$ is the first point in $D$ of the segment that goes from $x$ to $T_{+}^{*}(x)$. Then,

$$
\begin{aligned}
& \int_{\Omega}\left|x-T_{+}^{*}(x)\right| f^{+}(x) d x=\int_{\Omega}\left|x-\tilde{T}_{+}(x)\right| f^{+}(x) d x+\int_{\Omega}\left|\tilde{T}_{+}(x)-T_{+}^{*}(x)\right| f^{+}(x) d x \\
& =\int_{\Omega \times \Omega}|x-y| d\left(\left(I d \times \tilde{T}_{+}\right) \# f^{+}\right)(x, y)+\int_{\Omega \times \Omega}|x-y| d\left(\left(\tilde{T}_{+} \times T_{+}^{*}\right) \# f^{+}\right)(x, y) .
\end{aligned}
$$


If we define the measure $\tilde{h}_{\infty}:=\tilde{T}_{+} \# f^{+}$, which is supported on $\partial D$, we have that $\left(I d \times \tilde{T}_{+}\right) \# f^{+}$ is a transport plan induced by the map $\tilde{T}_{+}$between $f^{+}$and the measure $\tilde{h}_{\infty}$. On the other hand, since

$$
\begin{gathered}
\pi_{0} \#\left(\left(\tilde{T}_{+} \times T_{+}^{*}\right) \# f^{+}\right)(B)=\left(\tilde{T}_{+} \times T_{+}^{*}\right) \# f^{+}\left(\pi_{0}^{-1}(B)\right)=f^{+}\left(\left(\left(\tilde{T}_{+} \times T_{+}^{*}\right)\right)^{-1}\left(\pi_{0}^{-1}(B)\right)\right) \\
=f^{+}\left(\tilde{T}_{+}^{-1}(B)\right)=\tilde{T}_{+} \# f^{+}(B)=\tilde{h}_{\infty}(B) .
\end{gathered}
$$

and

$$
\begin{gathered}
\pi_{1} \#\left(\left(\tilde{T}_{+} \times T_{+}^{*}\right) \# f^{+}\right)(B)=\left(\tilde{T}_{+} \times T_{+}^{*}\right) \# f^{+}\left(\pi_{1}^{-1}(B)\right)=f^{+}\left(\left(\left(\tilde{T}_{+} \times T_{+}^{*}\right)\right)^{-1}\left(\pi_{1}^{-1}(B)\right)\right) \\
=f^{+}\left(T_{+}^{*-1}(B)\right)=T_{+}^{*} \# f^{+}(B)=h_{\infty}(B),
\end{gathered}
$$

we have that $\tilde{\gamma}(x, y):=\left(\left(\tilde{T}_{+} \times T_{+}^{*}\right) \# f^{+}\right)(x, y)$ is a transport plan between $\tilde{h}_{\infty}$ and $h_{\infty}$.

Now, by $(2.5),\left(I d \times \tilde{T}_{+}\right) \# f^{+}$is an optimal transport plan between $f^{+}$and $\tilde{h}_{\infty}$, and $\tilde{\gamma}$ is an optimal transport plan between $\tilde{h}_{\infty}$ and $h_{\infty}$.

By [1, Theorem 6.2], there exists an optimal transport map $\tilde{T}_{-}$transferring $f^{-}$to $\tilde{h}_{\infty}$. Let us see that $\left(\tilde{T}_{+}, \tilde{T}_{-}\right)$is a solution, for the matching problem, that is,

$$
\mathcal{F}\left(\tilde{T}_{+}, \tilde{T}_{-}\right)=\mathcal{F}\left(T_{+}^{*}, T_{-}^{*}\right)
$$

Indeed, if we consider the gluing transport of $\left(I d \times \tilde{T}_{-}\right) \# f_{-}$and $\hat{\gamma}(x, y):=\tilde{\gamma}(y, x)$, we have that

$$
\begin{gathered}
\int_{\Omega \times \Omega}|x-y| d\left(\left(I d \times T_{-}^{*}\right) \# f_{-}\right)(x, y)+\int_{\Omega \times \Omega}|x-y| d \hat{\gamma}(x, y) \\
\geq \int_{\Omega \times \Omega}|x-y| d\left(\left(I d \times \tilde{T}_{-}\right) \# f_{-}\right)(x, y) .
\end{gathered}
$$

Therefore,

$$
\begin{aligned}
& \mathcal{F}\left(T_{+}^{*}, T_{-}^{*}\right)=\int_{\Omega}\left|x-T_{+}^{*}(x)\right| f^{+}(x) d x+\int_{\Omega}\left|x-T_{-}^{*}(x)\right| f^{-}(x) d x \\
& =\int_{\Omega}\left|x-\tilde{T}_{+}(x)\right| f^{+}(x) d x+\int_{\Omega}\left|\tilde{T}_{+}(x)-T_{+}^{*}(x)\right| f^{+}(x) d x+\int_{\Omega}\left|x-T_{-}^{*}(x)\right| f^{-}(x) d x \\
& \quad \geq \int_{\Omega}|x-y| d\left(\left(I d \times \tilde{T}_{+}\right) \# f^{+}\right)(x, y)+\int_{\Omega \times \Omega}|x-y| d\left(\left(I d \times \tilde{T}_{-}\right) \# f_{-}\right)(x, y)=\mathcal{F}\left(\tilde{T}_{+}, \tilde{T}_{-}\right) .
\end{aligned}
$$

Which implies the equality (2.6). Moreover, we also have that the gluing transport of $(I d \times$ $\left.\tilde{T}_{-}\right) \# f_{-}$and $\hat{\gamma}$ is an optimal transport plan between $f^{+}$and $\tilde{h}_{\infty}$.

Remark 2.1. Let us remark that Theorem 1.1 is also true in the case that we change in the cost function the Euclidean norm by any norm in $\mathbb{R}^{N}$. In fact, we only need to have in mind that in this case Kantorovich potentials also exist (e.g., [17]), and the result in [4] that shows the existece of optimal transport maps $T_{+}^{*}$ transferring $f^{+}$to $\mu_{\infty}$ amd $T_{-}^{*}$ transferring $f^{-}$to $\mu_{\infty}$ 
Remark 2.2. Since

$$
\sup _{\substack{v, w \in W^{1, \infty}(\Omega) \\|\nabla v|_{\infty},|\nabla w|_{\infty} \leq 1}} \int_{\Omega} v\left(f^{+}-\mu\right)+w\left(f^{-}-\mu\right)=\sup _{\substack{v, w \in W^{1, \infty}(\Omega) \\|\nabla v|_{\infty},|\nabla w|_{\infty} \leq 1}} \int_{\Omega} v f^{+}-w f^{-}+(w-v) \mu
$$

and

$$
\begin{aligned}
\int_{\Omega} v f^{+}-w f^{-}+(w-v) \mu & =\int_{\Omega} v f^{+}-\left(w-\min _{D}(w-v)\right) f^{-}+\left(w-\min _{D}(w-v)-v\right) \mu \\
& \leq \sup _{\substack{\tilde{v}, \tilde{w} \in W^{1, \infty}(\Omega) \\
|\nabla \tilde{v}|_{\infty},|\nabla \tilde{w}|_{\infty} \leq 1 \\
\tilde{v} \leq \tilde{w} \text { in } D}} \int_{\Omega} \tilde{v} f^{+}-\tilde{w} f^{-}+(\tilde{w}-\tilde{v}) \mu,
\end{aligned}
$$

we have

$$
\sup _{\substack{v, w \in W^{1, \infty}(\Omega) \\|\nabla v|_{\infty},|\nabla w|_{\infty} \leq 1}} \int_{\Omega} v\left(f^{+}-\mu\right)+w\left(f^{-}-\mu\right)=\sup _{\substack{v, w \in W^{1, \infty}(\Omega) \\|\nabla v|_{\infty},|\nabla w|_{\infty} \leq 1 \\ v \leq w \text { in } D}} \int_{\Omega} v f^{+}-w f^{-}+(w-v) \mu .
$$

Therefore,

$$
\begin{gathered}
W=\inf _{\mu \in \mathcal{M}\left(D, M_{0}\right)} \sup _{\substack{v, w \in W^{1, \infty}(\Omega) \\
|\nabla v|_{\infty},|\nabla w|_{\infty} \leq 1 \\
v \leq w \text { in } D}} \int_{\Omega} v f^{+}-w f^{-}+(w-v) \mu \\
=\sup _{\substack{v, w \in W^{1, \infty}(\Omega) \\
|\nabla v|_{\infty},|\nabla w|_{\infty} \leq 1 \\
v \leq w \text { in } D}} \int_{\Omega} v f^{+}-w f^{-}+(w-v) \mu_{\infty} .
\end{gathered}
$$

If we could change inf sup by sup inf in the above expression, then we should get

$$
\begin{aligned}
& W=\inf _{\mu \in \mathcal{M}\left(D, M_{0}\right)} \sup _{\substack{v, w \in W^{1, \infty}(\Omega) \\
|\nabla v|_{\infty},|\nabla w|_{\infty} \leq 1 \\
v \leq w \text { in } D}} \int_{\Omega} v f^{+}-w f^{-}+(w-v) \mu \\
& =\sup _{\substack{v, w \in W^{1, \infty}(\Omega) \\
|\nabla v|_{\infty},|\nabla w|_{\infty} \leq 1 \\
v \leq w \text { in } D}} \inf _{\mu \in \mathcal{M}\left(D, M_{0}\right)} \int_{\Omega} v f^{+}-w f^{-}+(w-v) \mu=\sup _{\substack{v, w \in W^{1, \infty}(\Omega) \\
|\nabla v|_{\infty},|\nabla w|_{\infty} \leq 1 \\
v \leq w \text { in } D}} \int_{\Omega} v f^{+}-w f^{-},
\end{aligned}
$$

and hence we expect that

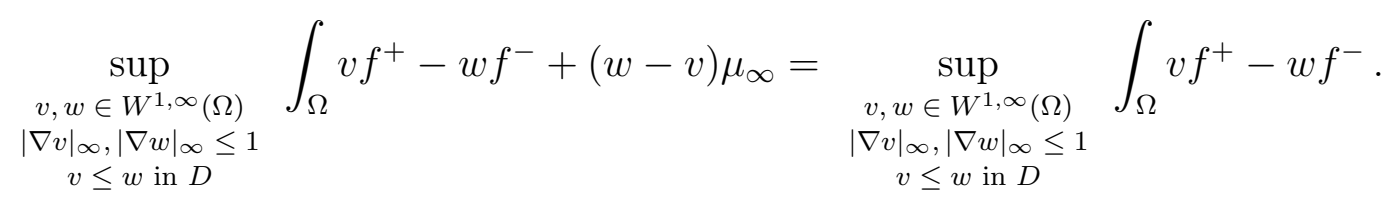


We will show that in fact the above equality is true, which is the key of our approach to the problem. The proof of this equality is based on the approximation of this problem by a $p$-Laplacian system (see the next section) and does not use min-max arguments.

\section{The Limit aS $p \rightarrow \infty$ IN A $p$-LAPlaCiAn SYSTEM}

In this section we show that we can follow the ideas of Evans-Gangbo, [12], to get the matching measure, and the Kantorovich potentials at the same time.

Take $p>N$ in this section and recall that, for simplicity, we assumed that $\Omega$ is a convex $C^{1,1}$ bounded open set.

First, we need some preliminary results.

Lemma 3.1 (A Poincaré's type inequality). There exists a constant $C>0$ such that

$$
\|(f, g)\|_{p} \leq C\left(\|(\nabla f, \nabla g)\|_{p}+\left|\int_{\Omega}(f+g)\right|\right)
$$

for all $(f, g) \in W^{1, p}(\Omega) \times W^{1, p}(\Omega), f \leq g$ in $D, f\left(x_{0}\right)=g\left(x_{0}\right)$ for some $x_{0} \in D$.

Proof. Let us argue by contradiction supposing that there exists $\left(f_{n}, g_{n}\right) \in W^{1, p}(\Omega) \times W^{1, p}(\Omega)$, $f_{n} \leq g_{n}$ in $D, f_{n}\left(x_{n}\right)=g_{n}\left(x_{n}\right)$ for some $x_{n} \in D$, such that

$$
\left\|\left(f_{n}, g_{n}\right)\right\|_{p} \geq n\left(\left\|\left(\nabla f_{n}, \nabla g_{n}\right)\right\|_{p}+\left|\int_{\Omega}\left(f_{n}+g_{n}\right)\right|\right) \text {. }
$$

By homogeneity we can suppose that

$$
\left\|\left(f_{n}, g_{n}\right)\right\|_{p}=1
$$

So,

$$
\left\|\left(\nabla f_{n}, \nabla g_{n}\right)\right\|_{p} \rightarrow 0
$$

and

$$
\int_{\Omega}\left(f_{n}+g_{n}\right) \rightarrow 0
$$

Therefore, there exists a subsequence, denoted equal, such that

$$
\left(f_{n}, g_{n}\right) \rightarrow(f, g) \text { uniformly and weakly in } W^{1, p}(\Omega) \times W^{1, p}(\Omega),
$$

and

$$
x_{n} \rightarrow x \in D, f(x)=g(x) .
$$

Now, by (3.2), $f$ and $g$ are constants, so equal. And by (3.3), $f=g=0$, which contradicts the fact that they come from the uniform limit of a subsequence satisfying (3.1).

The following consequence is immediate. 
Corollary 3.2. There exists $C>0$ such that

$$
\|(f, g)\|_{p} \leq C\|(\nabla f, \nabla g)\|_{p}
$$

for all $(f, g) \in W^{1, p}(\Omega) \times W^{1, p}(\Omega), f \leq g$ in $D, f\left(x_{0}\right)=g\left(x_{0}\right)$ for some $x_{0} \in D$, with

$$
\int_{\Omega}(f+g)=0
$$

Remark 3.3. The constants that appear in Lemma 3.1 and in Corollary 3.2 may depend on $p$. The method used in the proof does not allow to quantify such dependence (and it is not our aim in this paper to make this dependence precise), then we are not allowed to use these results in the passage to the limit as $p \rightarrow \infty$, they are used only to show existence and uniqueness of a solution of the elliptic system under consideration. To pass to the limit we rely on a local Morrey inequality, see the proof of Theorem 3.5 below.

Let us consider the following variational problem

$$
\min _{\substack{(v, w) \in W^{1, p}(\Omega) \times W^{1, p}(\Omega) \\ v \leq w \text { in } D}} \frac{1}{p} \int_{\Omega}|D v|^{p}+\frac{1}{p} \int_{\Omega}|D w|^{p}-\int_{\Omega} v f^{+}+\int_{\Omega} w f^{-} .
$$

Our next result in this section deals with existence and uniqueness of solutions for the variational problem (3.5).

Theorem 3.4. There exists a minimizer $\left(v_{p}, w_{p}\right)$ of (3.5). In addition any two minimizers differ by a constant, that is, if $\left(v_{p}, w_{p}\right)$ and $\left(\tilde{v}_{p}, \tilde{w}_{p}\right)$ are minimizers then there exists a constant $c$ with $v_{p}=\tilde{v}_{p}+c$ and $w_{p}=\tilde{w}_{p}+c$.

Proof. Set

$$
\Psi(v, w):=\frac{1}{p} \int_{\Omega}|D v|^{p}+\frac{1}{p} \int_{\Omega}|D w|^{p}-\int_{\Omega} v f^{+}+\int_{\Omega} w f^{-} .
$$

Let us begin by observing that, since the functions in $W^{1, p}(\Omega)$ are continuous, it is easy to see that

$$
\begin{array}{ccc}
\min _{\substack{(v, w) \in W^{1, p}(\Omega) \times W^{1, p}(\Omega) \\
v \leq w \text { in } D}} \Psi(v, w)= & \min _{(v, w) \in W^{1, p}(\Omega) \times W^{1, p}(\Omega)}^{v \leq w \text { in } D} \\
& \exists(v, w) . \\
& \exists x_{0} \in D, v\left(x_{0}\right)=w\left(x_{0}\right)
\end{array}
$$

Moreover, since

$$
\Psi(v, w)=\Psi(v-c, w-c) \text { for any constant } c
$$

by taking

$$
c=\frac{1}{2}\left(\frac{1}{|\Omega|} \int_{\Omega} v+\frac{1}{|\Omega|} \int_{\Omega} w\right)
$$


we can minimize $\Psi(v, w)$ between functions $(v, w)$ with

$$
\int_{\Omega} v+\int_{\Omega} w=0
$$

Now, by Corollary 3.2,

$$
\Psi(v, w):=\frac{1}{p} \int_{\Omega}|D v|^{p}+\frac{1}{p} \int_{\Omega}|D w|^{p}-\int_{\Omega} v f^{+}+\int_{\Omega} w f^{-}
$$

is a finite lower semicontinuous and coercive convex functional for the closed convex subset of $W^{1, p}(\Omega) \times W^{1, p}(\Omega), \mathcal{B}$, given by

$\left\{(v, w) \in W^{1, p}(\Omega) \times W^{1, p}(\Omega): v \leq w\right.$ in $D, v\left(x_{0}\right)=w\left(x_{0}\right)$ for some $\left.x_{0} \in D, \int_{\Omega}(v+w)=0\right\}$.

Then, by [2, Corollary 3.23], $\Psi$ attains its infimum on $\mathcal{B}$, which is equivalent to say that

$$
\inf _{\substack{(v, w) \in W^{1, p}(\Omega) \times W^{1, p}(\Omega) \\ v \leq w \text { in } D}} \Psi(v, w)
$$

is attained.

Finally, let us show uniqueness of the minimizer up to an additive constant. Equivalently, we prove uniqueness of the minimizer when we impose the constraint

$$
\int_{\Omega} v+\int_{\Omega} w=0
$$

Assume that we have two pairs $\left(v_{p}, w_{p}\right)$ and $\left(\tilde{v}_{p}, \tilde{w}_{p}\right)$ of minimizers and that

$$
\int_{\Omega} v_{p}+\int_{\Omega} w_{p}=\int_{\Omega} \tilde{v}_{p}+\int_{\Omega} \tilde{w}_{p}=0 .
$$

By the strict convexity of the function $\xi \mapsto\|\xi\|_{p}$ (we have $1<p<\infty$ ) we obtain that $D v_{p}=D \tilde{v}_{p}$ and $D w_{p}=D \tilde{w}_{p}$. Then there are constants $c_{1}$ and $c_{2}$ such that $v_{p}=\tilde{v}_{p}+c_{1}$ and $w_{p}=\tilde{w}_{p}+\tilde{c}_{2}$. Hence, from (3.7) we get that

$$
c_{1}+c_{2}=0 .
$$

Therefore, we obtain

$$
\Psi\left(v_{p}, w_{p}\right)=\Psi\left(\tilde{v}_{p}, \tilde{w}_{p}\right)-c_{1}\left(\int_{\Omega} f^{+}+\int_{\Omega} f^{-}\right)
$$

and we conclude that

$$
c_{1}=c_{2}=0
$$

from the fact that both pairs are minimizers.

Now we prove that we can pass to the limit as $p \rightarrow \infty$ in the sequence of minimizer functions. 
Theorem 3.5. Let $\left(v_{p}, w_{p}\right)$ be minimizer functions of (3.5). Then, up to a subsequence,

$$
\lim _{p \rightarrow \infty}\left(v_{p}, w_{p}\right)=\left(v_{\infty}, w_{\infty}\right) \quad \text { uniformly, }
$$

where $\left(v_{\infty}, w_{\infty}\right)$ is a solution of the variational problem

$$
\max _{\substack{v, w \in W^{1, \infty}(\Omega) \\|\nabla v|_{\infty},|\nabla w|_{\infty} \leq 1 \\ v \leq w \text { in } D}} \int_{\Omega} v f^{+}-w f^{-} .
$$

Remark 3.6. As we will see, the limit $\left(v_{\infty}, w_{\infty}\right)$ gives a pair of Kantorovich potentials for our optimal matching problem. But in fact this limit procedure gives much more since it allows us to identify the optimal matching measure (see Theorem 3.11 below).

Proof of Theorem 3.5. Let us take $\left(v_{p}, w_{p}\right) \in \mathcal{B}$ a minimizer of $(3.5)$. For $(v, w) \in W^{1, \infty}(\Omega) \times$ $W^{1, \infty}(\Omega)$, with $|\nabla v|_{\infty},|\nabla w|_{\infty} \leq 1$ and $v \leq w$ in $D$, we have that

$$
\begin{aligned}
-\int_{\Omega} v_{p} f^{+}+\int_{\Omega} w_{p} f^{-} & \leq \frac{1}{p} \int_{\Omega}\left|D v_{p}\right|^{p}+\frac{1}{p} \int_{\Omega}\left|D w_{p}\right|^{p}-\int_{\Omega} v_{p} f^{+}+\int_{\Omega} w_{p} f^{-} \\
& \leq \frac{1}{p} \int_{\Omega}|D v|^{p}+\frac{1}{p} \int_{\Omega}|D w|^{p}-\int_{\Omega} v f^{+}+\int_{\Omega} w f^{-} \\
& \leq 2 \frac{|\Omega|}{p}-\int_{\Omega} v f^{+}+\int_{\Omega} w f^{-} .
\end{aligned}
$$

Now, by (3.6), we can assume that there exists $x_{p} \in D$ such that $v_{p}\left(x_{p}\right)=w_{p}\left(x_{p}\right)$. We can also assume that $v_{p}\left(z_{\infty}\right)=0$ for all $p$, for any $z_{\infty} \in \Omega$. Hence, as $p>N$, we have:

$$
\left\|v_{p}\right\|_{\infty} \leq C_{1}\left\|D v_{p}\right\|_{p}
$$

and

$$
\left\|w_{p}\right\|_{\infty} \leq C_{1}\left(\left\|D w_{p}\right\|_{p}+\left\|D v_{p}\right\|_{p}\right),
$$

with $C_{1}$ not depending on $p$. Indeed, for a fixed $x \in \Omega$, there exists $x=x_{0}, x_{1}, \ldots, x_{m}=z_{\infty}$ and $m$ balls $Q_{i}(i=1,2, \ldots, m)$ of certain fixed diameter $r>0$, such that $x_{i}, x_{i+1} \in Q_{i+1}$ and $r \cdot m$ is bounded independently of $x, z_{\infty}$ and $m$. Then, local Morrey's inequality (see, e.g., the Remark in page 268 of [10] or [2]), implies

$$
\left|v_{p}(x)\right|=\left|v_{p}(x)-v_{p}\left(z_{\infty}\right)\right| \leq \sum_{i=1}^{m}\left|v_{p}\left(x_{i}\right)-v_{p}\left(x_{i+1}\right)\right| \leq C_{0} r^{1-N / p} m\left\|\nabla v_{p}\right\|_{p} \leq C_{1}\left\|\nabla v_{p}\right\|_{p},
$$

being $C_{i}$ independent of $p$. With the same argument, but changing the extreme points and the function, we obtain

$$
\left|w_{p}(x)\right|=\left|w_{p}(x)-w_{p}\left(x_{p}\right)\right|+\left|v_{p}\left(x_{p}\right)\right| \leq \sum_{i=1}^{\tilde{m}}\left|w_{p}\left(y_{i}\right)-w_{p}\left(y_{i+1}\right)\right|+\left|v_{p}\left(x_{p}\right)\right|
$$




$$
\leq C_{0} r^{1-N / p} \tilde{m}\left\|\nabla w_{p}\right\|_{p}+\left|v_{p}\left(x_{p}\right)\right| \leq C_{1}\left\|\nabla w_{p}\right\|_{p}+\left|v_{p}\left(x_{p}\right)\right|
$$

From (3.9), using Hölder's inequality and having in mind (3.10) and (3.11), we get

$\frac{1}{p} \int_{\Omega}\left|D v_{p}\right|^{p}+\frac{1}{p} \int_{\Omega}\left|D w_{p}\right|^{p} \leq C_{2}\left(\left\|v_{p}\right\|_{L^{p}(\Omega)}+\left\|w_{p}\right\|_{L^{p}(\Omega)}+1\right) \leq C_{3}\left(\left\|D v_{p}\right\|_{L^{p}(\Omega)}+\left\|D w_{p}\right\|_{L^{p}(\Omega)}+1\right)$, with $C_{i}$ independent of $p$. Hence,

$$
\left\|\nabla v_{p}\right\|_{L^{p}(\Omega)}^{p-1},\left\|\nabla w_{p}\right\|_{L^{p}(\Omega)}^{p-1} \leq p C_{4} \quad \forall p>N
$$

with $C_{4}$ independent of $p$. ity

Therefore, $\left\|v_{p}\right\|_{W^{1, p}(\Omega)}$ and $\left\|w_{p}\right\|_{W^{1, p}(\Omega)}$ are bounded uniformly in $p$, and, by Morrey's inequal-

$$
\left\{\begin{array}{l}
\left|v_{p}(x)-v_{p}(y)\right| \leq C_{5}|x-y|^{1-\frac{N}{p}} \\
\left|w_{p}(x)-w_{p}(y)\right| \leq C_{5}|x-y|^{1-\frac{N}{p}}
\end{array}\right.
$$

for some constant $C_{5}$ not depending on $p$. Then, by Arzela-Ascoli's compactness criterion we can extract a sequence $p_{i} \rightarrow \infty$ such that

$$
\begin{gathered}
v_{p_{i}} \rightarrow v_{\infty} \quad \text { uniformly in } \bar{\Omega}, \\
w_{p_{i}} \rightarrow w_{\infty} \quad \text { uniformly in } \bar{\Omega},
\end{gathered}
$$

and, so, $v_{\infty} \leq w_{\infty}$ in $D$. Moreover, by (3.12), we have

$$
\left\|\nabla v_{\infty}\right\|_{\infty},\left\|\nabla w_{\infty}\right\|_{\infty} \leq 1
$$

Finally, passing to the limit in (3.9), we get

$$
\int_{\Omega} v_{\infty} f^{+}-w_{\infty} f^{-}=\sup _{\substack{v, w \in W^{1, \infty}(\Omega) \\|\nabla v|_{\infty},|\nabla w|_{\infty} \leq 1 \\ v \leq w \text { in D }}} \int_{\Omega} v f^{+}-w f^{-} .
$$

This ends the proof.

Remark 3.7. Remark that the convergence as $p \rightarrow \infty$ is only along a subsequence. The main content of our result is that there is enough compactness to pass to the limit along subsequences and moreover that all possible limits are solutions to the maximization limit problem.

We now prove some properties of the minimizers and their limits that show that we have found (in the limit) Kantorovich potentials and an optimal matching measure for our matching problem.

We divide the proof of these properties in a series of lemmas.

Lemma 3.8. Let $\left(v_{p}, w_{p}\right)$ be minimizer functions of problem (3.5). Then, there exists a positive Radon measure $h_{p}$ of mass $M_{0}$ such that 
(1) $\left\{\begin{array}{ll}-\Delta_{p} v_{p}=f^{+}-h_{p} & \text { in } \Omega, \\ \left|\nabla v_{p}\right|^{p-2} \nabla v_{p} \cdot \eta=0 & \text { on } \partial \Omega,\end{array} \quad \begin{cases}-\Delta_{p} w_{p}=h_{p}-f^{-} & \text {in } \Omega, \\ \left|\nabla w_{p}\right|^{p-2} \nabla w_{p} \cdot \eta=0 & \text { on } \partial \Omega .\end{cases}\right.$

(2) The positive measure $h_{p}$ is supported on $\left\{x \in D: v_{p}(x)=w_{p}(x)\right\}$.

Proof. Recall that since $p>N$, we have $W^{1, p}(\Omega) \subset C(\bar{\Omega})$. For any $\varphi, \psi \in W^{1, p}(\Omega)$ such that $\varphi=\psi$ in $D$, since $\left(v_{p}, w_{p}\right)$ is a minimizer of $\Psi$ in the set

$$
\left\{(v, w) \in W^{1, p}(\Omega) \times W^{1, p}(\Omega): v \leq w \text { in } D\right\},
$$

the function

$$
I_{1}(t):=\Psi\left(v_{p}+t \varphi, w_{p}+t \psi\right)
$$

has a minimum at $t=0$. Therefore, $I_{1}^{\prime}(0)=0$, from where it follows that

$$
\int_{\Omega}\left|\nabla v_{p}\right|^{p-2} \nabla v_{p} \nabla \varphi+\int_{\Omega}\left|\nabla w_{p}\right|^{p-2} \nabla w_{p} \nabla \psi=\int_{\Omega} f^{+} \varphi-\int_{\Omega} f^{-} \psi .
$$

Observe that, taking $\psi=\varphi$ in (3.13), we get that

$$
\begin{cases}-\Delta_{p} v_{p}-\Delta_{p} w_{p}=f^{+}-f^{-} & \text {in } \Omega, \\ \left|\nabla v_{p}\right|^{p-2} \nabla v_{p} \cdot \eta+\left|\nabla w_{p}\right|^{p-2} \nabla w_{p} \cdot \eta=0 & \text { on } \partial \Omega .\end{cases}
$$

Similarly, for any $\varphi \in W^{1, p}(\Omega), \varphi \geq 0$, and any $t>0$, we have

$$
I_{2}(t):=\Psi\left(v_{p}-t \varphi, w_{p}\right)-\Psi\left(v_{p}, w_{p}\right) \geq 0
$$

and

$$
I_{3}(t):=\Psi\left(v_{p}, w_{p}+t \varphi\right)-\Psi\left(v_{p}, w_{p}\right) \geq 0 .
$$

Then, by taking limits in $\frac{I_{i}(t)}{t} i=2,3$, as $t \rightarrow 0$, we get

$$
\begin{cases}-\Delta_{p} v_{p} \leq f^{+} & \text {in } \Omega, \\ -\Delta_{p} w_{p} \geq-f^{-} & \text {in } \Omega .\end{cases}
$$

Therefore, $h_{p}:=\Delta_{p} v_{p}+f^{+}$defines a positive measure which, thanks to (3.14), is equal to $f^{-}-\Delta_{p} w_{p}$. The fact that $h_{p}$ is supported on $\left\{x \in D: v_{p}(x)=w_{p}(x)\right\}$ follows from the fact that, for $\varphi \in \mathcal{D}(\Omega)$ supported on $\Omega \backslash\left\{x \in D: v_{p}(x)=w_{p}(x)\right\}$ and $t \neq 0$ small enough,

$$
I_{4}(t):=\Psi\left(v_{p}+t \varphi, w_{p}\right)-\Psi\left(v_{p}, w_{p}\right) \geq 0 .
$$

Again, by taking limits in $\frac{I_{4}(t)}{t}$ as $t \rightarrow 0$, we conclude. This gives the proof of (2).

Given $\varphi \in \mathcal{D}\left(\mathbb{R}^{N}\right)$, if we take $\psi \in \mathcal{D}(\Omega)$ such that $\varphi=\psi$ en $D,(3.13)$ says that

$$
\int_{\Omega}\left|\nabla v_{p}\right|^{p-2} \nabla v_{p} \nabla \varphi+\int_{\Omega}\left|\nabla w_{p}\right|^{p-2} \nabla w_{p} \nabla \psi=\int_{\Omega} f^{+} \varphi-\int_{\Omega} f^{-} \psi .
$$


But, since $\psi \in \mathcal{D}(\Omega)$ and $\operatorname{supp}\left(h_{p}\right) \subset D$, we have

$$
\int_{\Omega}\left|\nabla w_{p}\right|^{p-2} \nabla w_{p} \nabla \psi=\int_{\Omega} \psi d h_{p}-\int_{\Omega} f^{-} \psi=\int_{\Omega} \varphi d h_{p}-\int_{\Omega} f^{-} \psi
$$

Then, from the two above expressions, by density we obtain that

$$
\int_{\Omega}\left|\nabla v_{p}\right|^{p-2} \nabla v_{p} \nabla \varphi=\int_{\Omega} f^{+} \varphi-\int_{\Omega} \varphi d h_{p}, \quad \forall \varphi \in W^{1, p}(\Omega),
$$

which shows the first statement in (1) for the first problem. Similarly, we obtain the second one. From here, now, it is an easy consequence that (just take $\varphi=1$ in (3.15))

$$
\int_{\Omega} d h_{p}=M_{0}
$$

and the proof concludes.

Lemma 3.9. Under the same assumptions of Lemma 3.8, up to a subsequence,

$$
h_{p} \rightarrow h_{\infty} \text { as } p \rightarrow \infty \text {, weakly* as measures, }
$$

with $h_{\infty}$ a positive Radon measure of mass $M_{0}$ supported on $\left\{x \in D: v_{\infty}(x)=w_{\infty}(x)\right\}$. And the limit $\left(v_{\infty}, w_{\infty}\right)$ obtained in Theorem 3.5 satisfies:

$$
\begin{aligned}
& v_{\infty} \text { is a Kantorovich potential for the transport of } f^{+} \text {to } h_{\infty} \text {, } \\
& w_{\infty} \text { is a Kantorovich potential for the transport of } h_{\infty} \text { to } f^{-} \text {, }
\end{aligned}
$$

with respect to the Euclidean distance.

Proof. From the last equality in the proof of the previous lemma,

$$
\int_{\Omega} d h_{p}=M_{0}
$$

we can assume that there exists a positive Radon measure $h_{\infty}$ of mass $M_{0}$ such that, up to a subsequence,

$$
h_{p} \rightarrow h_{\infty}
$$

Let $\varphi \in \mathcal{D}(\Omega)$ be supported on $\Omega \backslash\left\{x \in D: v_{\infty}(x)=w_{\infty}(x)\right\}$. Then, since

$$
\lim _{p}\left(v_{p}, w_{p}\right)=\left(v_{\infty}, w_{\infty}\right) \quad \text { uniformly, }
$$

there exists $p_{0}>N$ such that $\varphi$ is supported on $\Omega \backslash\left\{x \in D: v_{p}(x)=w_{p}(x)\right\}$ for all $p \geq p_{0}$. Therefore,

$$
\int_{\Omega} \varphi d h_{\infty}=\lim _{p \rightarrow \infty} \int_{\Omega} \varphi d h_{p}=0
$$

Consequently, $h_{\infty}$ is supported on $\left\{x \in D: v_{\infty}=w_{\infty}\right\}$.

Since $|\xi|^{p}-|\eta|^{p} \leq p|\xi|^{p-2} \xi \cdot(\xi-\eta)$ for any $\xi, \eta \in \mathbb{R}^{N}$, we have

$$
\frac{1}{p} \int_{\Omega}\left|\nabla v_{p}\right|^{p}-\int_{\Omega}\left(f^{+}-d h_{p}\right) v_{p}+\int_{\Omega}\left|\nabla v_{p}\right|^{p-2} \nabla v_{p} \cdot\left(\nabla \varphi-\nabla v_{p}\right)-\int_{\Omega}\left(f^{+}-d h_{p}\right)\left(\varphi-v_{p}\right)
$$




$$
\leq \frac{1}{p} \int_{\Omega}|\nabla \varphi|^{p}-\int_{\Omega}\left(f^{+}-d h_{p}\right) \varphi
$$

for every $\varphi \in W^{1, p}(\Omega)$. Then, having in mind (3.15), we have

$$
\left.\int_{\Omega}\left|\nabla v_{p}\right|^{p-2} \nabla v_{p} \cdot\left(\nabla \varphi-\nabla v_{p}\right)-\int_{\Omega}\left(f^{+}-d h_{p}\right)\left(\varphi-v_{p}\right)\right)=0,
$$

and we arrive to

$$
\frac{1}{p} \int_{\Omega}\left|\nabla v_{p}\right|^{p}-\int_{\Omega}\left(f^{+}-d h_{p}\right) v_{p} \leq \frac{1}{p} \int_{\Omega}|\nabla \varphi|^{p}-\int_{\Omega}\left(f^{+}-d h_{p}\right) \varphi \quad \forall \varphi \in W^{1, p}(\Omega) .
$$

Therefore, for any $v \in W^{1, \infty}(\Omega),|\nabla v|_{\infty} \leq 1$,

$$
\begin{aligned}
-\int_{\Omega}\left(f^{+}-d h_{p}\right) v_{p} & \leq \frac{1}{p} \int_{\Omega}\left|\nabla v_{p}\right|^{p}-\int_{\Omega}\left(f^{+}-d h_{p}\right) v_{p} \\
& \leq \frac{1}{p} \int_{\Omega}|\nabla v|^{p}-\int_{\Omega}\left(f^{+}-d h_{p}\right) v \leq \frac{1}{p}|\Omega|-\int_{\Omega}\left(f^{+}-d h_{p}\right) v .
\end{aligned}
$$

Taking limit as $p \rightarrow \infty$ in the last inequality, we get

$$
\int_{\Omega}\left(f^{+}-d h_{\infty}\right) v \leq \int_{\Omega}\left(f^{+}-d h_{\infty}\right) v_{\infty}
$$

from where it follows that

$$
\int_{\Omega}\left(f^{+}-d h_{\infty}\right) v_{\infty}=\sup _{\substack{v \in W^{1, \infty}(\Omega) \\|\nabla v|_{\infty} \leq 1}} \int_{\Omega} v\left(f^{+}-d h_{\infty}\right)
$$

and consequently, $v_{\infty}$ is a Kantorovich potential for the transport of $f^{+}$to $h_{\infty}$, with respect to the Euclidean distance. The proof for $w_{\infty}$ is similar.

Lemma 3.10. $\left(v_{\infty}, w_{\infty}\right)$ and $h_{\infty}$ satisfy

$$
\begin{aligned}
\int_{\Omega} v_{\infty} f^{+}-w_{\infty} f^{-}=\inf _{\mu \in \mathcal{M}\left(D, M_{0}\right)} \sup _{\substack{v, w \in W^{1, \infty}(\Omega) \\
|\nabla v|_{\infty},|\nabla w|_{\infty} \leq 1 \\
v \leq w \text { in } D}} \int_{\Omega} v f^{+}-w f^{-}+(w-v) \mu \\
=\min _{\left(\gamma_{+}, \gamma_{-}\right) \in \Pi_{D}\left(f^{+}, f^{-}\right)}\left\{\int_{\Omega \times \Omega}|x-y| d \gamma_{+}+\int_{\Omega \times \Omega}|x-y| d \gamma_{-}\right\} \\
=\inf _{\left(T_{+}, T_{-}\right) \in \mathcal{A}_{D}\left(f^{+}, f^{-}\right)}\left|x \int_{\Omega}\right| x-T_{+}(x)\left|f^{+}(x) d x+\int_{\Omega}\right| x-T_{-}(x) \mid f^{-}(x) d x .
\end{aligned}
$$

Proof. From Lemma 3.9 we have

$$
\int_{\Omega} v_{\infty}\left(f^{+}-d h_{\infty}\right)=\inf _{\mu \in \mathcal{A}\left(f^{+}, h_{\infty}\right)} \int|x-y| d \mu=\int|x-y| d \mu_{0},
$$


$\mu_{0} \in \mathcal{A}\left(f^{+}, h_{\infty}\right)$ and

$$
\int_{\Omega} w_{\infty}\left(d h_{\infty}-f^{-}\right)=\inf _{\nu \in \mathcal{A}\left(h_{\infty}, f^{-}\right)} \int|x-y| d \nu=\int|x-y| d \nu_{0},
$$

$\nu_{0} \in \mathcal{A}\left(h_{\infty}, f^{-}\right)$. Then, by adding the above inequalities and since $\int_{\Omega}\left(w_{\infty}-v_{\infty}\right) h_{\infty}=0$, we get

$$
\begin{aligned}
& \int_{\Omega} v_{\infty} f^{+}-w_{\infty} f^{-} \\
& =\int_{\Omega} v_{\infty} f^{+}-w_{\infty} f^{-}+\left(w_{\infty}-v_{\infty}\right) d h_{\infty}=\int|x-y| d \mu_{0}+\int|x-y| d \nu_{0} \\
& \geq \inf _{\left(\gamma_{+}, \gamma_{-}\right) \in \Pi_{D}\left(f^{+}, f^{-}\right)}\left\{\int_{\Omega \times \Omega}|x-y| d \gamma_{+}+\int_{\Omega \times \Omega}|x-y| d \gamma_{-}\right\} \\
& =\sup _{\substack{v, w \in W^{1, \infty}(\Omega) \\
|\nabla v|_{\infty},|\nabla w|_{\infty} \leq 1 \\
v \leq w \text { in } D}} \int_{\Omega} v f^{+}-w f^{-}+(w-v) \mu_{\infty} \\
& \geq \sup _{\substack{v, w \in W^{1, \infty}(\Omega) \\
|\nabla v|_{\infty},|\nabla w|_{\infty} \leq 1 \\
v \leq w \text { in } D}} \int_{\Omega} v f^{+}-w f^{-},
\end{aligned}
$$

where $\mu_{\infty}$ is the optimal measure given in Section 2 .

Theorem 3.11. The measure $h_{\infty}$ is a matching measure to the optimal matching problem (1.1).

Proof. From Lemma 3.9, there exists a positive measure $h_{\infty}$ and Lipschitz continuous functions $v_{\infty}, w_{\infty}$ satisfying:

$$
\begin{aligned}
& v_{\infty} \text { is a Kantorovich potential for the transport of } f^{+} \text {to } h_{\infty}, \\
& w_{\infty} \text { is a Kantorovich potential for the transport of } h_{\infty} \text { to } f^{-} \text {, }
\end{aligned}
$$

with respect to the Euclidean distance. Moreover, $h_{\infty}$ is supported on $\left\{x \in D: v_{\infty}=w_{\infty}\right\}$. Then, by [1, Theorem 2.1] and Kantorovich-Rubinstein Theorem (e.g. [17, Theorem 1.14]), we have

$$
\int_{\Omega}\left|x-T_{+}^{*}(x)\right| f^{+}(x) d x=\min \left\{\int_{\Omega \times \Omega}|x-y| d \gamma(x, y): \gamma \in \Pi\left(f^{+}, h_{\infty}\right)\right\}=\int_{\Omega} v_{\infty}\left(f^{+}-h_{\infty}\right)
$$

and

$$
\int_{\Omega}\left|y-T_{-}^{*}(x)\right| f^{-}(x) d x=\min \left\{\int_{\Omega \times \Omega}|x-y| d \gamma(x, y): \gamma \in \Pi\left(h_{\infty}, f^{-}\right)\right\}=\int_{\Omega} w_{\infty}\left(h_{\infty}-f^{-}\right) .
$$


Therefore, having in mind (3.17), we get

$$
\begin{gathered}
\inf _{\left(T_{+}, T_{-}\right) \in \mathcal{A}_{D}\left(f^{+}, f^{-}\right)} \int_{\Omega}\left|x-T_{+}(x)\right| f^{+}(x) d x+\int_{\Omega}\left|x-T_{-}(x)\right| f^{-}(x) d x \\
=\int_{\Omega}\left|x-T_{+}^{*}(x)\right| f^{+}(x) d x+\int_{\Omega}\left|y-T_{-}^{*}(x)\right| f^{-}(x) d x,
\end{gathered}
$$

which finishes the proof.

Observe that the above result gives an alternative proof for the first statement in Theorem 1.1. We will see in Theorem 3.14 that in some cases this approach also selects a matching measure supported on the boundary of the target set, which is the second statement of Theorem 1.1.

Remark 3.12. Note that, for the measure $\mu_{\infty}$ given in Section 2, it also holds that

$$
\int_{\Omega}\left(w_{\infty}-v_{\infty}\right) \mu_{\infty}=0
$$

which implies that $\mu_{\infty}$ is, in fact, supported where $v_{\infty}=w_{\infty}$ in $D$. Indeed, by (3.18),

$$
\begin{gathered}
\int_{\Omega} v_{\infty} f^{+}-w_{\infty} f^{-}=\sup _{\substack{v, w \in W^{1, \infty}(\Omega) \\
|\nabla v|_{\infty},|\nabla w|_{\infty} \leq 1 \\
v \leq w \text { in } D}} \int_{\Omega} v f^{+}-w f^{-}+(w-v) \mu_{\infty} \\
\geq \int_{\Omega} v_{\infty} f^{+}-w_{\infty} f^{-}+\left(w_{\infty}-v_{\infty}\right) \mu_{\infty} \geq \int_{\Omega} v_{\infty} f^{+}-w_{\infty} f^{-},
\end{gathered}
$$

which implies (3.19).

Lemma 3.13. If $\left(v_{p}, w_{p}\right)$ is a pair solving the equations in Lemma 3.8 for a positive measure $h_{p}$, and

$$
\begin{gathered}
v_{p} \leq w_{p} \quad \text { in } D, \\
\operatorname{supp}\left(h_{p}\right) \subset\left\{x \in D: v_{p}(x)=w_{p}(x)\right\},
\end{gathered}
$$

then, $\left(v_{p}, w_{p}\right)$ is a minimizer in the minimization problem (3.5).

Proof. We have that

$$
\frac{1}{p} \int_{\Omega}\left|\nabla v_{p}\right|^{p}-\int_{\Omega}\left(f^{+}-d h_{p}\right) v_{p} \leq \frac{1}{p} \int_{\Omega}|\nabla \varphi|^{p}-\int_{\Omega}\left(f^{+}-d h_{p}\right) \varphi \quad \forall \varphi \in W^{1, p}(\Omega),
$$

and

$$
\frac{1}{p} \int_{\Omega}\left|\nabla w_{p}\right|^{p}-\int_{\Omega}\left(d h_{p}-f^{-}\right) w_{p} \leq \frac{1}{p} \int_{\Omega}|\nabla \psi|^{p}-\int_{\Omega}\left(d h_{p}-f^{-}\right) \psi \quad \forall \psi \in W^{1, p}(\Omega) .
$$

Adding up both expressions, since $h_{p}$ is supported in $D$ where $v_{p}=w_{p}$, and $v_{p} \leq w_{p}$ in $D$,

$$
\frac{1}{p} \int_{\Omega}\left|\nabla v_{p}\right|^{p}+\frac{1}{p} \int_{\Omega}\left|\nabla w_{p}\right|^{p}-\int_{\Omega} f^{+} v_{p}+\int_{\Omega} f^{-} w_{p}
$$




$$
\leq \frac{1}{p} \int_{\Omega}|\nabla \varphi|^{p}+\frac{1}{p} \int_{\Omega}|\nabla \psi|^{p}-\int_{\Omega} f^{+} \varphi+\int_{\Omega} f^{-} \psi
$$

for all $\varphi, \psi \in W^{1, p}(\Omega), \varphi \leq \psi$ in $D$.

Theorem 3.14. Assume that $D$ is the closure of a smooth domain $\Theta$, then $h_{p}$ is supported on $\partial D$ and hence $h_{\infty}$ is concentrated on the boundary of $D$.

Proof. Let $\tilde{v}_{p}, \tilde{w}_{p}$ be minimizers of

$$
\min _{\substack{(v, w) \in W^{1, p}(\Omega) \times W^{1, p}(\Omega) \\ v \leq w \text { in } \partial D}} \Psi(v, w) .
$$

and let $\tilde{h}_{p}$ be a positive measure, $\operatorname{supp}\left(\tilde{h}_{p}\right) \subset\left\{x \in \partial D: \tilde{v}_{p}(x)=\tilde{w}_{p}(x)\right\}$, such that

$$
\left\{\begin{array}{l}
-\Delta_{p} \tilde{v}_{p}=f^{+}-\tilde{h}_{p} \quad \text { in } \Omega, \\
\left|\nabla \tilde{v}_{p}\right|^{p-2} \nabla \tilde{v}_{p} \cdot \eta=0
\end{array} \quad \text { on } \partial D, \quad \begin{cases}-\Delta_{p} \tilde{w}_{p}=\tilde{h}_{p}-f^{-} & \text {in } \Omega, \\
\left|\nabla \tilde{w}_{p}\right|^{p-2} \nabla \tilde{w}_{p} \cdot \eta=0 & \text { on } \partial D .\end{cases}\right.
$$

Set now $\bar{v}_{p}=\tilde{v}_{p}$ in $\Omega \backslash D$, and define $\bar{v}_{p}$ in $D$ as the solution of

$$
\begin{cases}-\Delta_{p} v=0 & \text { in } \Theta \\ v=\tilde{v}_{p} & \text { on } \partial D\end{cases}
$$

Similarly we define $\bar{w}_{p}$. Observe that, by the the Maximum Principle

$$
\bar{v}_{p} \leq \bar{w}_{p} \text { in } D
$$

and also

$$
\int_{\Theta}\left|D \bar{v}_{p}\right|^{p} \leq \int_{\Theta}\left|D \tilde{v}_{p}\right|^{p}, \quad \int_{\Theta}\left|D \bar{w}_{p}\right|^{p} \leq \int_{\Theta}\left|D \tilde{w}_{p}\right|^{p}
$$

Then,

$$
\begin{aligned}
& \Psi\left(\bar{v}_{p}, \bar{w}_{p}\right)=\frac{1}{p} \int_{\Omega}\left|D \bar{v}_{p}\right|^{p}+\frac{1}{p} \int_{\Omega}\left|D \bar{w}_{p}\right|^{p}-\int_{\Omega} \bar{v}_{p} f^{+}+\int_{\Omega} \bar{w}_{p} f^{-} \\
& =\frac{1}{p} \int_{\Omega \backslash D}\left|D \tilde{v}_{p}\right|^{p}+\frac{1}{p} \int_{\Theta}\left|D \bar{v}_{p}\right|^{p}+\frac{1}{p} \int_{\Omega \backslash D}\left|D \tilde{w}_{p}\right|^{p}+\frac{1}{p} \int_{\Theta}\left|D \bar{w}_{p}\right|^{p}-\int_{\Omega} \tilde{v}_{p} f^{+}+\int_{\Omega} \tilde{w}_{p} f^{-} \\
& \leq \frac{1}{p} \int_{\Omega \backslash D}\left|D \tilde{v}_{p}\right|^{p}+\frac{1}{p} \int_{\Theta}\left|D \tilde{v}_{p}\right|^{p}+\frac{1}{p} \int_{\Omega \backslash D}\left|D \tilde{w}_{p}\right|^{p}+\frac{1}{p} \int_{\Theta}\left|D \tilde{w}_{p}\right|^{p}-\int_{\Omega} \tilde{v}_{p} f^{+}+\int_{\Omega} \tilde{w}_{p} f^{-} \\
& =\Psi\left(\tilde{v}_{p}, \tilde{w}_{p}\right) .
\end{aligned}
$$

In fact, since $\bar{v}_{p} \leq \bar{w}_{p}$ in $\partial D$,

$$
\Psi\left(\bar{v}_{p}, \bar{w}_{p}\right)=\Psi\left(\tilde{v}_{p}, \tilde{w}_{p}\right)
$$


Hence, by Theorem 3.4, there exists a constant $c$ such that $\left(\bar{v}_{p}, \bar{w}_{p}\right)=\left(\tilde{v}_{p}+c, \tilde{w}_{p}+c\right)$, and consequently,

$$
\left\{\begin{array} { l l } 
{ - \Delta _ { p } \overline { v } _ { p } = f ^ { + } - \tilde { h } _ { p } } & { \text { in } \Omega , } \\
{ | \nabla \overline { v } _ { p } | ^ { p - 2 } \nabla \overline { v } _ { p } \cdot \eta = 0 } & { \text { on } \partial \Omega , }
\end{array} \quad \left\{\begin{array}{ll}
-\Delta_{p} \bar{w}_{p}=\tilde{h}_{p}-f^{-} & \text {in } \Omega, \\
\left|\nabla \bar{w}_{p}\right|^{p-2} \nabla \bar{w}_{p} \cdot \eta=0 & \text { on } \partial \Omega .
\end{array}\right.\right.
$$

Then, since $\bar{v}_{p} \leq \bar{w}_{p}$ in $D$, by Lemma 3.13, we have $\left(\bar{v}_{p}, \bar{w}_{p}\right)$ is a minimizer of Problem (3.5). Therefore, by Theorem 3.4, there exists a constant $\bar{c}$ such that $\left(\bar{v}_{p}, \bar{w}_{p}\right)=\left(v_{p}+\bar{c}, w_{p}+\bar{c}\right)$, and consequently, $h_{p}=\tilde{h}_{p}$, which implies that $h_{\infty}$ is supported on $\partial D$.

\section{EXAMPLES}

Let us first compute some examples that illustrate our results and next characterize when the optimal matching measure is a delta.

Example 4.1. Consider the optimal matching problem for the data: $\Omega=]-4,4\left[, f^{+}=\right.$ $b \chi_{]-3,-2[}+(1-b) \chi_{] 2,3[}, f^{-}=\chi_{]-2,-1[}$ and $D=[0,1]$, where $0 \leq b \leq 1$ is fixed. Then, any matching measure in $D$ is of the form $b \delta_{0}+\mu$, for any positive Radon measure $\mu$, of mass $1-b$, supported on $D$. Indeed, it is easy to see that, for

$$
T_{+}^{*}(x)= \begin{cases}0 & \text { if }-3<x<-2 \\ t_{+}^{*}(x) & \text { in other case }\end{cases}
$$

where $t_{+}^{*}$ is any optimal transport map transporting $(1-b) \chi_{] 2,3[}$ to $\mu$, and

$$
T_{-}^{*}(x)= \begin{cases}0 & \text { if }-2<x<-2+b \\ t_{-}^{*}(x) & \text { in other case }\end{cases}
$$

where $t_{-}^{*}$ is any optimal transport map transporting $\chi_{]-2+b,-1[}$ to $\mu$,

$$
\mathcal{F}\left(T_{+}^{*}, T_{-}^{*}\right)=4 .
$$

Also, for

$$
v^{*}(x):= \begin{cases}-x & \text { if } x \leq 0 \\ x & \text { if } x \geq 0\end{cases}
$$

and

$$
\begin{gathered}
w^{*}(x)=x \\
\int_{\Omega} v^{*}(x) f^{+}(x) d x-w^{*}(x) f^{-}(x) d x=4 .
\end{gathered}
$$


Then, our assertion follows from

$$
\begin{aligned}
\int_{\Omega} v^{*}(x) f^{+}(x) d x-w^{*}(x) f^{-}(x) d x & \\
\leq & \sup _{\substack{v, w \in W^{1, \infty}(\Omega) \\
|\nabla v|_{\infty},|\nabla w|_{\infty} \leq 1 \\
v \leq w \text { in } D}} \int_{\Omega} v f^{+}-w f^{-} \\
& =\inf _{\substack{\left(T_{+}, T_{-}\right) \in \mathcal{A}_{D}\left(f^{+}, f^{-}\right)\\
}} \mathcal{F}\left(T_{+}, T_{-}\right) \leq \mathcal{F}\left(T_{+}^{*}, T_{-}^{*}\right) .
\end{aligned}
$$

Observe also that, in this case, the cost for the usual transport of $f^{+}$to $f^{-}$is $(b-2)^{2}$.

We distinguish three cases:

1. If $b=1, \delta_{0}$ is the unique matching measure.

2. If $0<b<1$, there are infinitely many matching measures but all of them with singular part.

3. If $b=0$, we also have infinitely many matching measures, infinitely many without singular part and infinitely many with singular part. Moreover, only in this case, the cost of the matching problem is the same as the cost of the classical transport problem of $f^{+}$to $f^{-}$.

So we can not expect uniqueness of $h_{\infty}$ in general, but it may hold for some special configurations of the masses and the target set. Uniqueness of $h_{\infty}$ holds in one-dimension if and only if the target set $D$ is located to the left or to the right from the supports of $f^{+}$and $f^{-}$, while if there is some mass of $f^{+}$to the left of $D$ and some mass of $f^{-}$to the right (or viceversa) then there are infinitely many optimal measures $h_{\infty}$.

Moreover, in one dimension there is necessarily a singular part in the optimal measure $h_{\infty}$ if the masses $f_{+}$and $f_{-}$has some part of both of them to the left or to the right of $D$, while if $f_{+}$is completely on the right and $f_{-}$completely on the left of $D$ then there are optimal $h_{\infty}$ without singular part.

Now, let us come back to the symmetric situation given in the case $b=0$. In this case we can also compute optimal pairs $\left(v_{p}, w_{p}\right)$. Let

$$
z_{p}(x)=\frac{p-1}{p}|x|^{\frac{1}{p-1}} x
$$

This antisymmetric function $z_{p}$ is a solution to $-\left(\left|z^{\prime}\right|^{p-2} z^{\prime}\right)^{\prime}(x)=-1$ for $x>0$ with $z^{\prime}(0)=0$. Note that $\left(z_{p}\right)^{\prime}(1)=1$ and $z_{p}(1)=\frac{p-1}{p}$. Also note that

$$
z_{p}(x) \rightarrow x \quad \text { as } p \rightarrow \infty
$$


With the aid of this $z_{p}$ let us define $v_{p, c}$ and $w_{p, c}$ as follows. For any $c \in[0,1]$ we consider the functions

and

$$
v_{p, c}(x)=\left\{\begin{array}{lr}
0, & -4 \leq x \leq 0, \\
c x, & 0 \leq x \leq 1, \\
x+c-1, & 1 \leq x \leq 2, \\
z_{p}(x-3)+\frac{2 p-1}{p}+c, & 2 \leq x \leq 3, \\
\frac{2 p-1}{p}+c, & 3 \leq x \leq 4,
\end{array}\right.
$$

$$
w_{p, c}(x)=\left\{\begin{array}{lr}
-\frac{2 p-1}{p}, & -4 \leq x \leq-2, \\
z_{p}(x+2)-\frac{2 p-1}{p}, & -2 \leq x \leq-1, \\
x, & -1 \leq x \leq 0, \\
c x, & 0 \leq x \leq 1, \\
c, & 1 \leq x \leq 4
\end{array}\right.
$$

A simple computation gives

$$
-\left(\left|\left(v_{p, c}\right)^{\prime}\right|^{p-2}\left(v_{p, c}\right)^{\prime}\right)^{\prime}=f^{+}-\left(c^{p-1} \delta_{0}+\left(1-c^{p-1}\right) \delta_{1}\right)
$$

and

$$
-\left(\left|\left(w_{p, c}\right)^{\prime}\right|^{p-2}\left(w_{p, c}\right)^{\prime}\right)^{\prime}=\left(1-c^{p-1}\right) \delta_{0}+c^{p-1} \delta_{1}-f^{-} .
$$

Hence, taking

$$
c=\left(\frac{1}{2}\right)^{\frac{1}{p-1}},
$$

if we define $v_{p}:=v_{p, c}$ and $w_{p}:=w_{p, c}$, we have

$$
-\Delta_{p} v_{p}=f^{+}-h_{p} \quad \text { and } \quad-\Delta_{p} v_{p}=h_{p}-f^{-},
$$

being $h_{p}:=\frac{1}{2} \delta_{0}+\frac{1}{2} \delta_{1}$. Moreover $v_{p} \leq w_{p}$ in $D$ and $h_{p}$ is supported on $\left\{x \in D: v_{p}(x)=w_{p}(x)\right\}$. Therefore we have obtained a sequence of minimizers $\left(v_{p}, w_{p}\right)$ that gives in the limit the matching measure $\frac{1}{2} \delta_{0}+\frac{1}{2} \delta_{1}$. In addition it can be checked that the optimal Kantorovich potentials that appear in this limit procedure are just given by

$$
v_{\infty}(x)=\left\{\begin{array}{cc}
0, & -4 \leq x \leq 0 \\
x, & 0 \leq x \leq 3 \\
3, & 3 \leq x \leq 4
\end{array}\right.
$$

and

$$
w_{\infty}(x)=\left\{\begin{array}{lr}
-2, & -4 \leq x \leq-2 \\
x, & -2 \leq x \leq 1 \\
1, & 1 \leq x \leq 4
\end{array}\right.
$$


Note that $\left(v_{p}, w_{p}\right)$ is unique, up to a constant, that is, any other minimizer is of the form $\left(v_{p}+c, w_{p}+c\right), c$ constant. Therefore, this example shows that not every possible optimal matching measure can be obtained using this procedure.

Let us characterize now, in any space dimension, the set of configurations for which the matching measure is a delta concentrated at a point $z_{0} \in D$.

Theorem 4.2. Assume that there is a point $z_{0} \in D$ such that for any pair of points $x \in X_{+}$ and $y \in X_{-}$we have

$$
\min _{z \in D}\{|x-z|+|y-z|\}=\left|x-z_{0}\right|+\left|y-z_{0}\right|
$$

then the measure $M_{0} \delta_{z_{0}}$ is an optimal matching measure.

Conversely, if $M_{0} \delta_{z_{0}}$ is an optimal matching measure, then for any pair of points $x \in X_{+}$and $y \in X_{-}$we have (4.1).

Proof. Let $\hat{a}(x):=\left|x-z_{0}\right|$ for $x \in X_{+}$and $\hat{b}(x)=-\left|x-z_{0}\right|$ for $x \in X_{-}$. Both are 1-Lipschitz functions.

Let now $a(x):=\sup _{y \in X_{+}}\{\hat{a}(x)-|x-y|\}$ for $x \in \Omega$, and $b(x):=\inf _{y \in X_{-}}\{\hat{b}(x)+|x-y|\}$ for $x \in \Omega$, the lower 1 -Lipschitz extension of $a$ to $\Omega$ and the upper 1 -Lipschitz extension of $b$ to $\Omega$, respectively (in fact these are the McShane and Whitney extensions, see [15, 19]).

Let us see that $a \leq b$ on $D$. By (4.1) we have that, for $z \in D$,

$$
\left|x-z_{0}\right|-|x-z| \leq-\left|y-z_{0}\right|+|y-z| \quad \forall x \in X_{+} \text {and } \forall y \in X_{-} ;
$$

therefore, taking the supremum in $x$ and the infimum in $y$ we get that $a(z) \leq b(z)$.

Let us see now that $(a, b)$ is a maximizer of $(3.8)$. Let $(v, w)$ a pair of test functions, then

$$
v(x) \leq v\left(z_{0}\right)+\left|x-z_{0}\right| \quad \forall x \in X_{+}
$$

and

$$
w(y) \geq w\left(z_{0}\right)-\left|y-z_{0}\right| \quad \forall y \in X_{-} .
$$

Therefore, using that $v \leq w$ in $D$, we get

$$
\begin{gathered}
\int_{\Omega} v f^{+}-\int_{\Omega} w f^{-} \leq\left(v\left(z_{0}\right)-w\left(z_{0}\right)\right) \int_{\Omega} f^{+}+\int_{\Omega}\left|x-z_{0}\right| f^{+}(x) d x+\int_{\Omega}\left|y-z_{0}\right| f^{-}(y) d y \\
\leq \int_{\Omega}\left|x-z_{0}\right| f^{+}(x) d x+\int_{\Omega}\left|y-z_{0}\right| f^{-}(y) d y=\int_{\Omega} a f^{+}-\int_{\Omega} b f^{-} .
\end{gathered}
$$

Observe now that, setting $T_{+}^{*}(x)=z_{0}$ for $x \in X_{+}$and $T_{-}^{*}(x)=z_{0}$ for $x \in X_{-}$,

$$
\int_{\Omega} a f^{+}-\int_{\Omega} b f^{-}=\int_{\Omega}\left|x-T_{+}^{*}(x)\right| f^{+}(x) d x+\int_{\Omega}\left|y-T_{-}^{*}(y)\right| f^{-}(y) d y .
$$

Therefore, $M_{0} \delta_{z_{0}}$ is an optimal matching measure. 
To see the converse we argue by contradiction. Hence, assume that $M_{0} \delta_{z_{0}}$ is an optimal matching measure and that there are two points $x_{0} \in X_{+}$and $y_{0} \in X_{-}$such that (4.1) does not hold, that is, there exists $z_{1} \in D$ such that

$$
\left|x_{0}-z_{1}\right|+\left|y_{0}-z_{1}\right|<\left|x_{0}-z_{0}\right|+\left|y_{0}-z_{0}\right| \text {. }
$$

By continuity we can find a positive number $\eta$ and two small radii $r_{1}$ and $r_{2}$ such that

$$
\left|x-z_{1}\right|+\left|y-z_{1}\right|<\left|x-z_{0}\right|+\left|y-z_{0}\right|-\eta,
$$

for every $x \in B_{r_{1}}\left(x_{0}\right)$ and every $y \in B_{r_{2}}\left(y_{0}\right)$ and such that

$$
\int_{B_{r_{1}}\left(x_{0}\right)} f_{+}(x) d x=\int_{B_{r_{2}}\left(y_{0}\right)} f_{-}(y) d y=k>0 .
$$

Note that, thanks to this mass balance condition (4.3), we have an optimal transport map $x=S(y)$ that sends $f_{-} \chi_{B_{r_{2}}\left(y_{0}\right)}$ to $f_{+} \chi_{B_{r_{1}}\left(x_{0}\right)}$. In particular $S$ satisfies

$$
\int_{B_{r_{1}}\left(x_{0}\right)} A(x) f_{+}(x) d x=\int_{B_{r_{2}}\left(y_{0}\right)} A(S(y)) f_{-}(y) d y
$$

for every continuous function $A$. Hence,

$$
\int_{B_{r_{1}}\left(x_{0}\right)}\left|x-z_{i}\right| f^{+}(x) d x=\int_{B_{r_{2}}\left(y_{0}\right)}\left|S(y)-z_{i}\right| f^{-}(y) d y, \quad i=0,1
$$

and using (4.2), we obtain that

$$
\begin{aligned}
& \int_{B_{r_{1}}\left(x_{0}\right)}\left|x-z_{1}\right| f^{+}(x) d x+\int_{B_{r_{2}}\left(y_{0}\right)}\left|y-z_{1}\right| f^{-}(y) d y \\
& =\int_{B_{r_{2}}\left(y_{0}\right)}\left(\left|S(y)-z_{1}\right|+\left|y-z_{1}\right|\right) f^{-}(y) d y \\
& \leq \int_{B_{r_{2}}\left(y_{0}\right)}\left(\left|S(y)-z_{0}\right|+\left|y-z_{0}\right|\right) f^{-}(y) d y-k \eta \\
& =\int_{B_{r_{1}}\left(x_{0}\right)}\left|x-z_{0}\right| f^{+}(x) d x+\int_{B_{r_{2}}\left(y_{0}\right)}\left|y-z_{0}\right| f^{-}(y) d y-k \eta .
\end{aligned}
$$

Now let us define

$$
\tilde{T}_{+}(x)=\left\{\begin{array}{ll}
z_{0}, & x \in X_{+} \backslash B_{r_{1}}\left(x_{0}\right), \\
z_{1}, & x \in B_{r_{1}}\left(x_{0}\right),
\end{array} \quad \text { and } \quad \tilde{T}_{-}(y)= \begin{cases}z_{0}, & y \in X_{-} \backslash B_{r_{2}}\left(y_{0}\right) \\
z_{1}, & y \in B_{r_{2}}\left(y_{0}\right) .\end{cases}\right.
$$


This pair corresponds to the transport of $f_{+}$and $f_{-}$to the measure $\left(M_{0}-k\right) \delta_{z_{0}}+k \delta_{z_{1}}$ that is supported in $D$. We have

$$
\begin{aligned}
& \int_{\Omega}\left|x-\tilde{T}_{+}(x)\right| f^{+}(x) d x+\int_{\Omega}\left|y-\tilde{T}_{-}(y)\right| f^{-}(y) d y \\
& =\int_{X_{+} \backslash B_{r_{x}}\left(x_{0}\right)}\left|x-z_{0}\right| f^{+}(x) d x+\int_{X_{-} \backslash B_{r_{y}}\left(y_{0}\right)}\left|y-z_{0}\right| f^{-}(y) d y \\
& \quad+\int_{B_{r_{x}}\left(x_{0}\right)}\left|x-z_{1}\right| f^{+}(x) d x+\int_{B_{r_{y}}\left(y_{0}\right)}\left|y-z_{1}\right| f^{-}(y) d y \\
& <\int_{X_{+}}\left|x-z_{0}\right| f^{+}(x) d x+\int_{X_{-}}\left|y-z_{0}\right| f^{-}(y) d y-k \eta,
\end{aligned}
$$

a contradiction with the fact that $M_{0} \delta_{z_{0}}$ is an optimal matching measure.

Let us compute a more geometrical form of the condition (4.1) in case that $D$ is convex.

Assume that (4.1) holds, then differentiation of $|x-z|+|y-z|$ with respect to $z$ gives that $z_{0} \in D$ satisfies

$$
\left\langle\frac{x-z_{0}}{\left|x-z_{0}\right|}+\frac{y-x_{0}}{\left|y-z_{0}\right|}, z-z_{0}\right\rangle \leq 0 \quad \text { for all } x \in X_{+}, y \in X_{-} \text {and } z \in D
$$

(note that $z_{0}$ may belong to $\partial D$ ). Hence (4.1) implies (4.4).

The converse also holds: if $z_{0} \in D$ satisfies (4.4) then (4.1) holds. Indeed, arguing by contradiction, suppose that for some $z \in D$, we have,

$$
|x-z|+|y-z|<\left|x-z_{0}\right|+\left|y-z_{0}\right| .
$$

Now, by convexity of $z \mapsto|x-z|+|y-z|$, for $0<t<1$,

$$
\begin{aligned}
& \left|x-\left(t z+(1-t) z_{0}\right)\right|+\left|y-\left(t z+(1-t) z_{0}\right)\right| \\
& \leq t(|x-z|+|y-z|)+(1-t)\left(\left|x-z_{0}\right|+\left|y-z_{0}\right|\right) \\
& =t\left(|x-z|+|y-z|-\left|x-z_{0}\right|-\left|y-z_{0}\right|\right)+\left|x-z_{0}\right|+\left|y-z_{0}\right|,
\end{aligned}
$$

that is

$$
\begin{gathered}
\frac{1}{t}\left(\left|x-\left(t z+(1-t) z_{0}\right)\right|+\left|y-\left(t z+(1-t) z_{0}\right)\right|-\left|x-z_{0}\right|-\left|y-z_{0}\right|\right) \\
\leq|x-z|+|y-z|-\left|x-z_{0}\right|-\left|y-z_{0}\right| .
\end{gathered}
$$

Therefore, taking limit as $t \rightarrow 0$, and using (4.5),

$$
-\left\langle\frac{x-z_{0}}{\left|x-z_{0}\right|}+\frac{y-z_{0}}{\left|y-z_{0}\right|}, z-z_{0}\right\rangle \leq|x-z|+|y-z|-\left|x-z_{0}\right|-\left|y-z_{0}\right|<0,
$$

which gives a contradiction. 
Remark 4.3. Since we know that the target set in this problem can be reduced to the boundary, it is worth to search for a $z_{0} \in \partial D$ such that, for any pair of points $x \in X_{+}$and $y \in X_{-}$,

$$
\min _{z \in \partial D}\{|x-z|+|y-z|\}=\left|x-z_{0}\right|+\left|y-z_{0}\right|
$$

which also ensures the existence of a matching measure $M_{0} \delta_{z_{0}}$, now concentrated on the boundary of $D$.

\section{EXTENSIONS}

With the same ideas we can also consider the situation in which the cost is different for the transport of $f^{+}$to the set $D$ and for $f^{-}$to the set $D$. In fact we can consider the following cost functional

$$
\int_{\Omega} \frac{1}{A}\left|x-T_{+}(x)\right| f^{+}(x) d x+\int_{\Omega} \frac{1}{B}\left|x-T_{-}(x)\right| f^{-}(x) d x .
$$

With the constants $A$ and $B$ we are taking into account that the cost of transporting nuts and screws can be different (for example due to a difference in the weight).

For this kind of problems we only have to modify the $p$-Laplacian approximation replacing the $L^{p}$-norm of the gradient with

$$
\frac{1}{p} \int A^{p}|D v|^{p}
$$

In fact, doing this we are lead to consider variational problems of the form

$$
\min _{\substack{(v, w) \in W^{1, p}(\Omega) \times W^{1, p}(\Omega) \\ v \leq w \text { in } D}} \frac{1}{p} \int_{\Omega} A^{p}|D v|^{p}+\frac{1}{p} \int_{\Omega} B^{p}|D w|^{p}-\int_{\Omega} v f^{+}+\int_{\Omega} w f^{-},
$$

and when we pass to the limit as $p \rightarrow \infty$ we arrive to

$$
\max _{\substack{v, w \in W^{1, \infty}(\Omega) \\ A|\nabla v|_{\infty}, B|\nabla w|_{\infty} \leq 1 \\ v \leq w \text { in D }}} \int_{\Omega} v f^{+}-w f^{-} .
$$

Note that the constraint $A|\nabla v|_{\infty}, B|\nabla w|_{\infty} \leq 1$ is equivalent to

$$
|v(x)-v(y)| \leq \frac{1}{A}|x-y|, \quad|w(x)-w(y)| \leq \frac{1}{B}|x-y| .
$$

Hence we find Kantorovich potentials for the optimal matching problem of minimizing

$$
\int_{\Omega} \frac{1}{A}\left|x-T_{+}(x)\right| f^{+}(x) d x+\int_{\Omega} \frac{1}{B}\left|x-T_{-}(x)\right| f^{-}(x) d x .
$$

Another possible extension is the following. We can consider a matching problem with more than two commodities. Let $f^{1}, f^{2}, \ldots, f^{n}$, be nonnegative functions with the same total mass, 
that is,

$$
\int f^{i}=M_{0}
$$

for every $i$. Given a target set $D$ we can look at the minimization problem

$$
\min _{\left(T_{i}\right) \in \mathcal{A}_{D}} \sum_{i=1}^{n} \int_{\Omega}\left|x-T_{i}(x)\right| f^{i}(x) d x .
$$

where

$$
\begin{array}{r}
\mathcal{A}_{D}:=\left\{\left(T_{i}\right): T_{i}: \Omega \rightarrow \Omega \text { are Borel functions, } T_{i}\left(\operatorname{supp}\left(f^{i}\right)\right) \subset D,\right. \\
\left.\int_{T_{i}^{-1}(E)} f^{i}=\int_{T_{j}^{-1}(E)} f^{j} \text { for all Borel subset } E \text { of } \Omega\right\} .
\end{array}
$$

To handle this situation, say for three commodities, the minimization problem to take into account is given by

$$
\min _{\substack{(v, w, z) \in\left(W^{1, p}(\Omega)\right)^{3} \\ v+w+z \leq 0 \text { in } D}} \frac{1}{p} \int_{\Omega}|D v|^{p}+\frac{1}{p} \int_{\Omega}|D w|^{p}+\frac{1}{p} \int_{\Omega}|D z|^{p}-\int_{\Omega} v f^{1}-\int_{\Omega} w f^{2}-\int_{\Omega} z f^{3} .
$$

Note that this is similar to what we did before since (3.5) can be rewritten as

$$
\min _{\substack{(v, w) \in W^{1, p}(\Omega) \times W^{1, p}(\Omega) \\ v+w \leq \operatorname{in~} D}} \frac{1}{p} \int_{\Omega}|D v|^{p}+\frac{1}{p} \int_{\Omega}|D w|^{p}-\int_{\Omega} v f^{+}-\int_{\Omega} w f^{-} .
$$

We presented the details for only two masses since this simpler case shows how to handle the main mathematical difficulties.

Remark 5.1. One can try to solve the optimal matching problem for the Euclidean distance by taking the optimal matching measures for the cost $|x-y|^{r}$ with $r>1$ (these are uniformly convex costs) and then take the limit as $r \rightarrow 1$. This passage to the limit seems delicate and hence we preferred to perform instead the $p$-Laplacian approximation since it gives us not only the optimal matching measure but also gives the Kantorovich potentials.

Acknowledgements. Part of this work was performed during visits of the second author to U. Valencia. He wants to thank for the fruitful working atmosphere found there. The first and third authors have been partially supported by the Spanish Ministerio de Economía y Competitividad and FEDER, project MTM2012-31103. The second author is partially supported by the Spanish Ministerio de Economía y Competitividad under grants MTM2010-18128 and MTM2011-27998. 


\section{REFERENCES}

[1] L. Ambrosio. Lecture notes on optimal transport problems. Mathematical aspects of evolving interfaces (Funchal, 2000), 1-52, Lecture Notes in Math., 1812, Springer, Berlin, 2003.

[2] H. Brezis. Functional Analysis, Sobolev Spaces and Partial Differential Equations.Universitext, Springer, New York, 2011.

[3] G. Carlier. Duality and existence for a class of mass transportation problems and economic applications. Adv. Math. Econ. 5, 1-21, (2003).

[4] T. Champion and L. De Pascale. The Monge problem in $\mathbb{R}^{d}$, Duke Math. J. 157 (2011), 551-572.

[5] P-A. Chiappori, R. McCann and L. Nesheim. Hedoniic prices equilibria, stable matching, and optimal transport: equivalence, topolgy, and uniqueness, Econ. Theory 42 (2010), 317-354.

[6] I. Ekeland. An Optimal matching problem, ESAIM COCV 11 (2005), 57-71.

[7] I. Ekeland. Existence, uniqueness and efficiency of equilibrium in hedonic markets with multidimensional types. Econ. Theory, 42 (2010), no. 2, 275-315.

[8] I. Ekeland. Notes on optimal transportation. Econ. Theory 42 (2010), no. 2, 437û459.

[9] I. Ekeland, J.J Hecckman and L. Nesheim. Identificacation and Estimates of Hedonic Models, Journal of Political Economy 112 (2004), S60-S109.

[10] L. C. Evans. Partial Differential Equations. Grad. Stud. Math. 19, Amer. Math. Soc., 1998.

[11] L. C. Evans. Partial differential equations and Monge-Kantorovich mass transfer. Current developments in mathematics, 1997 (Cambridge, MA), 65-126, Int. Press, Boston, MA, 1999.

[12] L. C. Evans and W. Gangbo. Differential equations methods for the Monge-Kantorovich mass transfer problem. Mem. Amer. Math. Soc., 137 (1999), no. 653.

[13] N. Igbida, J.M. Mazón, J.D. Rossi and J.J. Toledo. A Monge-Kantorovich mass transport problem for a discrete distance. J. Funct. Anal. 260 (2011), 3494-3534.

[14] L. V. Kantorovich. On the tranfer of masses, Dokl. Nauk. SSSR 37 (1942), 227-229.

[15] E. J. McShane, Extension of range of functions. Bull. Amer. Math. Soc. 40 (1934), 837-842.

[16] J.M. Mazón, J.D. Rossi and J.J. Toledo. An optimal transportation problem with a cost given by the euclidean distance plus import/export taxes on the boundary. Preprint

[17] C. Villani. Topics in Optimal Transportation. Graduate Studies in Mathematics. Vol. 58, 2003.

[18] C. Villani. Optimal transport. Old and new. Grundlehren der MathematischenWissenschaften (Fundamental Principles of Mathematical Sciences), vol. 338. Springer, Berlin (2009).

[19] H. Whitney, Analytic extension of diferentiable functions defined in a closed sets. Trans. Amer. Math. Soc. 36 (1934), 63-89.

J. M. MAZÓN

Departament d'Anàlisi Matemàtica, U. de València, Valencia, Spain

mazon@uv.es

J. D. Rossi

Departamento de Análisis Matemático, U. de Alicante, Alicante, Spain

AND

Departamento de Matemática, FCeyn, U. de Buenos Aires, Buenos Aires, Argentina

julio.rossi@ua.es

J. TOLEDO

Departament d'Anàlisi Matemàtica, U. de València, Valencia, Spain

toledojj@uv.es 